\title{
Monochorionic quadramniotic and triamniotic pregnancies following single embryo transfers: two case reports and a review of the literature
}

\author{
Sotirios H. Saravelos ${ }^{1}$. Ting Zhang ${ }^{2}$. Jacqueline Pui Wah Chung ${ }^{1} \cdot$ Lu-Ming Sun ${ }^{3}$. \\ Yun $\mathrm{Sun}^{2} \cdot$ Tin-Chiu $\mathrm{Li}^{1} \cdot \mathrm{Zi}$-Jiang $\mathrm{Chen}^{2}$
}

Received: 26 August 2015 / Accepted: 28 October 2015 /Published online: 12 November 2015

(C) The Author(s) 2015. This article is published with open access at Springerlink.com

\begin{abstract}
Purpose The purpose of this study is to report two cases of monozygotic quadruplet and triplet pregnancies following single embryo transfer (ET).

Methods A 29-year-old woman and a 34-year-old woman underwent ART treatment in two affiliated University based ART units. The first woman underwent ICSI with day 3 embryo biopsy for pre-implantation genetic diagnosis (PGD) followed by day 4 transfer, which resulted in a monochorionic quadramniotic (MCQA) quadruplet pregnancy. The second woman underwent conventional IVF with transfer of a single blastocyst, which resulted in a monochorionic triamniotic (MCTA) triplet pregnancy. Results The first patient underwent successful selective foetal reduction at $16+3$ and $17+4$ weeks of gestation. Two healthy twin girls were delivered by elective caesarean section at $35+$ 6 weeks of gestation. The second patient underwent successful selective foetal reduction at $14+1$ weeks of gestation. The
\end{abstract}

Both Sotirios H. Saravelos and Ting Zhang are first authors.

Capsule To our knowledge, these cases represent the first 32 case of viable MCQA pregnancy following single ET in the 33 world and the third case of a viableMCTA pregnancy follow- 34 ing conventional IVF with single ET.

Zi-Jiang Chen

chenzijiang@hotmail.com

1 Assisted Reproductive Technology Unit, Prince of Wales Hospital, The Chinese University of Hong Kong, Shatin, Hong Kong

2 Center for Reproductive Medicine, Shanghai Key Laboratory for Assisted Reproduction and Reproductive Genetics, Renji Hospital, School of Medicine, Shanghai Jiao Tong University, Shanghai 200135, China

3 Fetal Medicine Unit and Prenatal Diagnosis Center, Department of Obstetrics, Shanghai First Maternity and Infant Hospital, Tongji University School of Medicine, Shanghai, China remaining monochorionic diamniotic (MCDA) twins are well at the time of writing this article.

Conclusions To our knowledge, these cases represent the first case of viable MCQA pregnancy following single ET in the world and the third case of a viable MCTA pregnancy following conventional IVF with single ET. Several factors including blastocyst stage transfer and zona pellucida manipulation have been thought to contribute to monozygotic twinning in the context of ART. These two cases add to the growing literature of monozygotic multiple pregnancies following ART.

Keywords IVF · ICSI · Pre-implantation genetic diagnosis · Multiple pregnancy $\cdot$ Ultrasound

\section{Introduction}

The increase of multiple pregnancies has been a major talking point in the field of ART for many years. It concerns not only multiple pregnancies relating to multiple embryo transfers (ET) [1] but also monozygotic twinning relating to single ET [2]. The universal occurrence of monozygotic twining in spontaneous conceptions is estimated to be approximately $0.4-0.45 \%$ $[3,4]$. This is traditionally thought to be higher (2-12-fold) in the ART population [5-9], although a recent study from the Danish National Cohort has reported reassuringly similar rates $(0.3 \%)$ in both spontaneous and ART derived pregnancies [10].

Higher order monozygotic pregnancies, defined as $\geq 3$ foetuses resulting from a single embryo, are exceedingly rare. For example, the rate of monozygotic triplets, which affects approximately $4.5 \%$ of all triplet gestations, is thought to be less than $0.004 \%$ of all spontaneous conceptions [11-14]. The rate of spontaneous monozygotic quadruplets is even rarer, occurring in approximately 1 in 10-15 million pregnancies [15]. In fact, there have been only a handful of cases reported with 
ultrasound evidence in the literature worldwide [16-18]. These cases are so rare that there appear to be only 28 cases of documented identical quadruplets in the whole of the USA [15].

As of 2012, over 5 million babies have been born following ART treatment [19]. To date, there appear to be only 18 cases of higher order monozygotic pregnancies reported worldwide in the literature. However, many more may have occurred without been reported in the literature, making the true incidence of higher order monozygotic pregnancies in the ART context difficult to estimate. To our knowledge, there has been only one reported case of monozygotic quadruplet pregnancy following ART treatment, of which all embryos were nonviable at 9 weeks and subsequently were found to have a diagnosis of 46,XX,inv [9] (p11q13) on karyotype analysis [20]. In this article, we report two further cases of high order monozygotic pregnancies following ART: one monochorionic quadramniotic (MCQA) quadruplet pregnancy following ICSI and embryo biopsy for pre-implantation genetic diagnosis (PGD) and one monochorionic triamniotic (MCTA) triplet pregnancy following conventional IVF. To our knowledge, the former is the first case of viable MCQA pregnancy following single ET in the world, while the latter is the third reported case of a viable MCTA pregnancy following conventional IVF with single ET.

\section{Case 1}

A 29-year-old woman with 2 years of primary infertility resulting from male factor infertility (oligoasthenospermia) and abnormal karyotype [45,XY, rob [13, 14] (q10;q10)] underwent PGD-ICSI treatment at the Center for Reproductive Medicine, Renji Hospital, Shanghai. During her first ART treatment cycle, stimulation was performed with daily administration of 150 units of recombinant follicle stimulating hormone (rFSH) (N.V.Organon, Netherlands) according to a standardised long protocol with Triptorelin $0.05 \mathrm{mg}$ daily downregulation (Ferring, Germany). Following hCG trigger, 17 oocytes were collected, of which eight cleavage stage embryos were biopsied. Following fluorescent in situ hybridization (FISH), two normal euploid embryos were diagnosed, of which one was selected for transfer. The embryo selected had undergone single cell biopsy at the 10 cell stage and was subsequently transferred on day 4 .

The woman successfully conceived, as confirmed by a hCG level of $2610 \mathrm{IU} / \mathrm{L}$ on day 15 post-transfer. Routine two-dimensional (2D) transvaginal ultrasound (US) by a reproductive medicine specialist on day 29 following transfer reported a single gestational sac $(37 \times 21 \times 37 \mathrm{~mm})$ with three yolk sacs and three foetal poles, all with heart beats. The TVU was repeated by the same doctor on day 35 post-transfer reporting a single gestational sac $(52 \times 19 \times 40 \mathrm{~mm})$ with three viable foetuses with crown rump lengths (CRL) of 10.5, 8.1 and $10.0 \mathrm{~mm}$. The couple was informed about the increased maternal and foetal risks with this condition and were counselled about the possibility of a foetal reduction procedure.

After consideration, the couple decided to undergo foetal reduction at the prenatal diagnosis centre (PDC) of the First Maternal and Infant Hospital of Shanghai. At 13+2 weeks of the pregnancy, an abdominal US was performed by a foetal medicine specialist in fact revealed the presence of a MCQA pregnancy with four equal sized viable foetuses (CRL of 82, 83,82 and $82 \mathrm{~mm}$ ), all with nuchal translucency measurements within normal range. The woman subsequently underwent selective foetal reduction via use of radiofrequency ablation on two occasions, at $16+3$ and $17+4$ weeks of gestation. In the absence of any definitive evidence, reduction of only one foetus at a time was performed, with the assumption that it may reduce the risk of miscarriage in the remaining foetuses. The procedures were uncomplicated, and two healthy twin girls were delivered by elective Caesarean Section at $35+6$ weeks of gestation, weighing 2060 and $1985 \mathrm{~g}$. The placental specimen along with the two selectively reduced foetuses confirmed a MCQA pregnancy (Fig. 1).

\section{Case 2}

A 34-year-old lady with 7 years of secondary infertility due to tubal factor underwent conventional IVF treatment at the University ART unit, Prince of Wales Hospital, Hong Kong. She had a previous first trimester miscarriage in 2007 and a right ectopic pregnancy in 2008, at which time she had a salpingectomy. Subsequent investigations including a

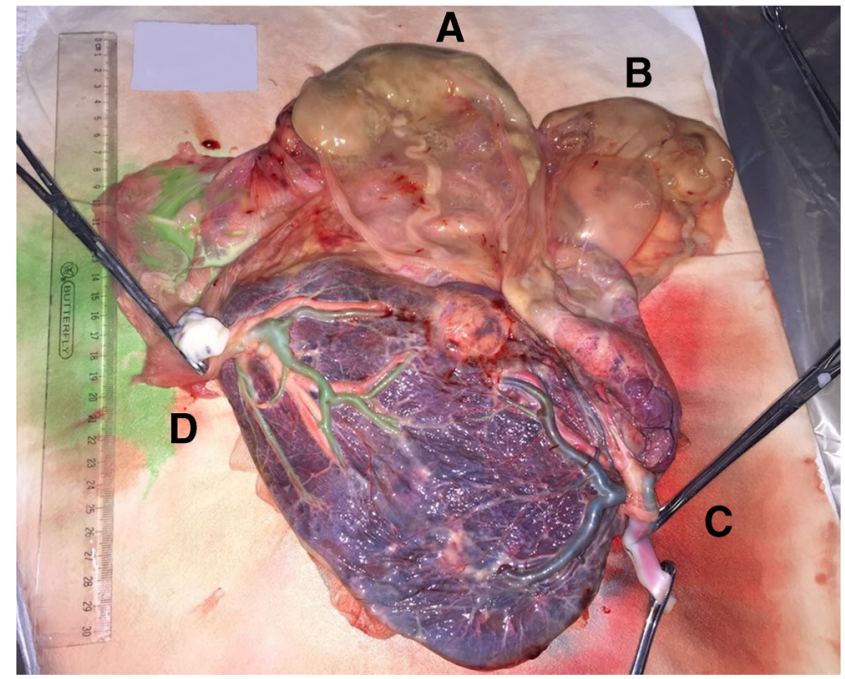

Fig. 1 Placental specimen at the time of delivery for case 1 confirming a MCQA pregnancy. $\mathbf{a}$ and $\mathbf{b}$ indicate the selectively reduced foetuses, still intact within their amniotic membranes and $\mathbf{c}$ and $\mathbf{d}$ the two umbilical cords of the two delivered baby girls. All cords were confirmed to be originating from a single placenta 
laparoscopy confirmed blockage of the contralateral tube and evidence of endometriosis. During her first ART cycle, stimulation was performed with daily administration of 225 units of human menopausal gonadotrophin (hMG) (Serono, Aubonne/Switzerland) according to a standardised antagonist protocol with Ganirelix $0.25 \mathrm{mg}$ downregulation (Merck Serono, Germany). Following hCG trigger, 14 mature oocytes were collected, of which 12 were fertilised, yielding 6 viable blastocysts (i.e. Gardner grading of BB grading or above) on day 5. No assisted hatching was performed, and a single expanding blastocyst (i.e. increasing blastocoel cavity with thinning of the zona pellucida and differentiation of the inner cell mass) was transferred to the patient on day 5 . The remaining blastocysts were all cryopreserved following appropriate consent.

The woman successfully conceived, as confirmed by an hCG level of $154 \mathrm{IU} / \mathrm{L}$ on day 9 post-transfer. Routine 2D transvaginal US 23 days following transfer was suspicious of monochorionic twin or triplet pregnancy; therefore, a 3D US was performed on the same day demonstrating clearly a single gestational sac with three yolk sacs and three foetal poles (CRL of 4.5, 3.1 and $3.0 \mathrm{~mm}$ ) (Fig. 2a). On day 36 following transfer, 3D US confirmed a MCTA pregnancy (Fig. 2b). The couple was informed appropriately regarding the increased maternal and foetal risks of higher order monozygotic gestations and was counselled regarding the possibility of a foetal reduction procedure.

After being reviewed by the foetal medicine team of the Prince of Wales Hospital, an US at $11+5$ weeks showed all three CRLs of the triplets to be within normal range, although the nuchal translucency was increased in one of the triplets ( $3.6 \mathrm{~mm}$ versus 2.3 and 1.2 for the other two). The couple subsequently decided to proceed with selective foetal reduction via use of radiofrequency ablation, which was successfully performed for the triplet with the thickest nuchal translucency at $14+1$ weeks of gestation. The procedure was uncomplicated, and the remaining twins are well at the time of writing this article.

\section{Discussion}

To our knowledge, the cases presented in this article represent the first viable MCQA pregnancy in the world resulting from ART and the third viable MCTA pregnancy resulting from conventional IVF with single blastocyst transfer. A systematic review of the literature shows that there are now a total of two monozygotic quadruplets and eighteen monozygotic triplets reported to date worldwide (Table 1). It is interesting to report these two present cases together, as they share many differences. The first case occurred following the transfer of a single day 4 embryo that had been fertilised by ICSI and biopsied for PGD, while the second case occurred following conventional

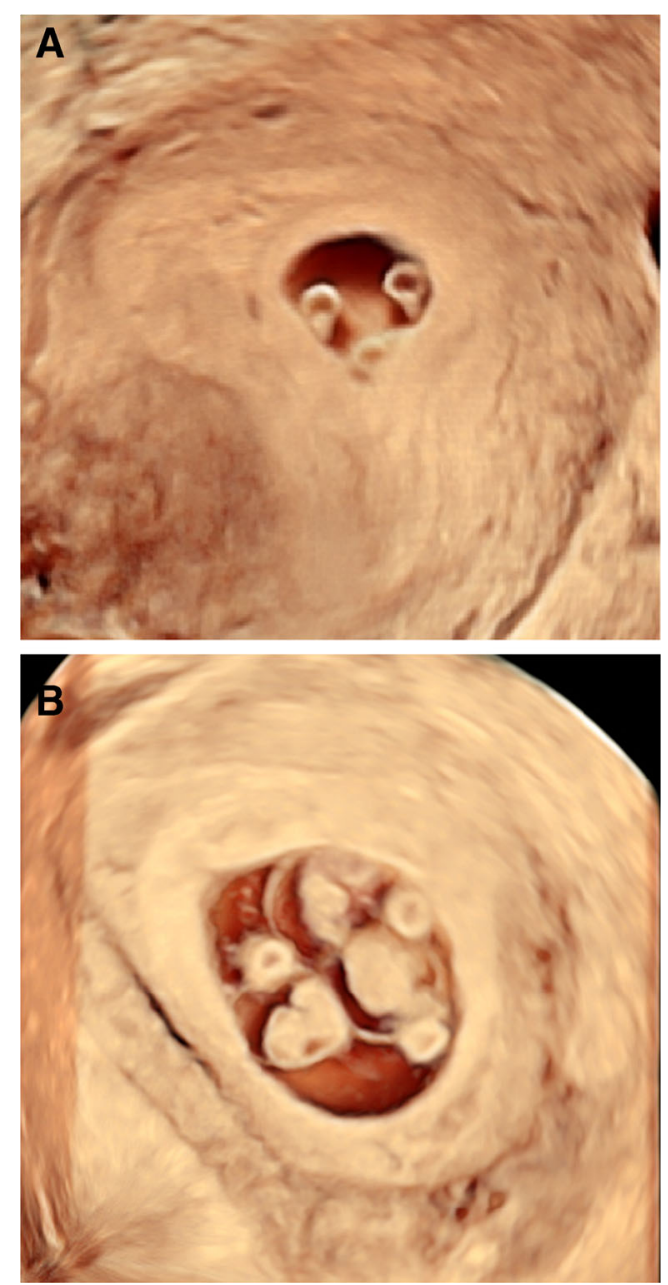

Fig. 2 3D US at a 23 days and b 36 days following transfer clearly demonstrating an MCTA pregnancy

IVF and transfer of a single blastocyst. Both therefore contribute to the growing literature of monozygotic higher order pregnancies following ART.

Historically, a potential association between monozygotic twinning and zona pellucida structure following ART was first proposed by Edwards et al. (1986) [5]. This was followed by a report of Alikani et al. (1994) on monozygotic twin pregnancies following breaching of the zona pellucida, and the proposal that the zona manipulation may affect the chance of monozygotic twinning [21]. This observation was followed by further publications supporting this hypothesis [22, 23]. The main mechanism suggested for this association is thought to be the herniation of blastomeres through the zona pellucida during blastocyst expansion, which may serve as a trigger for embryo splitting [24]. However, to date, whether this factor is indeed associated with an increased incidence of monozygotic multiple pregnancies remains debatable. Specifically, although there are a number of reports implying a correlation [21-23], others have failed to demonstrate a significant link [25-27]. This has led to some authors concluding that 
Table 1 Reports of high order $(\geq 3)$ monochorionic pregnancies following ART in the literature to date

\begin{tabular}{|c|c|c|c|c|c|c|c|c|}
\hline \multirow[t]{2}{*}{ Publication } & \multicolumn{3}{|c|}{ Zona pellucida breach } & \multirow[t]{2}{*}{ Embryonic stage } & \multirow{2}{*}{$\begin{array}{l}\text { No of } \\
\text { ET }\end{array}$} & \multirow{2}{*}{$\begin{array}{l}\text { Pregnancies } \\
\text { conceived }\end{array}$} & \multirow[t]{2}{*}{ Intervention } & \multirow[t]{2}{*}{ Outcome } \\
\hline & ICSI & $\begin{array}{l}\text { Assisted } \\
\text { hatching }\end{array}$ & $\begin{array}{l}\text { Embryo } \\
\text { biopsy }\end{array}$ & & & & & \\
\hline Salat-Baroux et al., 1994 & No & No & No & Cleavage & 4 & $\begin{array}{l}\text { MCTA triplets } \\
\text { DCDA twins }\end{array}$ & Reduction & DCDA miscarriage \\
\hline $\begin{array}{l}\text { Belaisch-Allart et al., } \\
1995\end{array}$ & No & No & No & Cleavage & 3 & MCTA triplets & Nil & Delivery ?weeks \\
\hline Yakin et al., 2001 & Yes & No & No & Blastocyst & 3 & $\begin{array}{l}\text { MCTA triplets } \\
\text { DCDA twins }\end{array}$ & Reduction & DCDA delivery 36 weeks \\
\hline Ghulmiyyah et al., 2003 & Yes & Yes & No & Blastocyst & 2 & MCTA triplets & Nil & $\mathrm{CS}$ at week 31 \\
\hline \multirow[t]{2}{*}{ Ulug et al., 2004} & Yes & Yes & No & Cleavage & 3 & $\begin{array}{l}\text { MCTA triplets } \\
\text { Singleton }\end{array}$ & Reduction & Singleton CS at week 38 \\
\hline & Yes & Yes & No & Cleavage & 3 & MCTA triplets & Nil & CS at week 34 \\
\hline Unger et al., 2004 & Yes & No & No & Blastocyst & 2 & $\begin{array}{l}\text { MCTA triplets } \\
\text { MCDA twins }\end{array}$ & Reduction & $\begin{array}{l}\text { Ongoing MCDA pregnancy } \\
22 \text { weeks }\end{array}$ \\
\hline Zikopoulos et al., 2004 & Yes & No & No & Blastocyst & 2 & $\begin{array}{l}\text { MCTA triplets } \\
\text { MCDA twins }\end{array}$ & Reduction & $\begin{array}{l}\text { Ongoing MCDA pregnancy } \\
20 \text { weeks }\end{array}$ \\
\hline Risquez et al., 2004 & Yes & Yes & No & Cleavage & 1 & MCTA triplets & Nil & $\begin{array}{l}\text { Ongoing pregnancy } \\
16 \text { weeks }\end{array}$ \\
\hline Jain et al., 2004 & No & No & No & Blastocyst & 2 & MCTA triplets & Nil & Ongoing pregnancy 7 weeks \\
\hline Henne et al., 2005 & No & No & No & Blastocyst & 2 & MCTA triplets & Termination & \\
\hline Yanaihara et al., 2007 & No & No & No & Blastocyst & 1 & MCTA triplets & Termination & \\
\hline Lee et al., 2008 & Yes & No & No & Blastocyst & 1 & MCTA triplets & Nil & $\mathrm{CS}$ at 33 weeks \\
\hline Faraj et al., 2008 & No & No & No & Blastocyst & 1 & MCTA triplets & Nil & $\mathrm{CS}$ at 32 weeks \\
\hline Pantos et al., 2004 & Yes & Yes & No & Day 4 & 3 & $\begin{array}{l}\text { MCTA triplets } \\
\text { singleton }\end{array}$ & Reduction & Singleton CS at 38 weeks \\
\hline $\begin{array}{l}\text { Haimov-Kochman } \\
\text { et al., } 2009\end{array}$ & Yes & No & Yes & Day 4 & 3 & $\begin{array}{l}\text { MCTA triplets } \\
\text { singleton }\end{array}$ & Reduction & Singleton delivery at 38 weeks \\
\hline Liu et al., 2010 & No & No & No & Cleavage & 2 & MCQA & Nil & Miscarriage \\
\hline Gurunath et al., 2015 & No & No & No & Blastocyst & 2 & MCTA & Termination & \\
\hline \multirow[t]{2}{*}{ Saravelos et al., 2015} & Yes & No & Yes & Day 4 & 1 & MCQA & Reduction & MCDA CS at $35+6$ weeks \\
\hline & No & No & No & Blastocyst & 1 & MCTA & Reduction & Ongoing MCDA pregnancy \\
\hline
\end{tabular}

$E T$ embryos transferred, $C S$ caesarean section, $D C D A$ dichorionic diamniotic, $M C D A$ monochorionic diamniotic, $M C T A$ monochorionic triamniotic

increased reporting of monozygotic twinning in this context may simply reflect the increasing use of procedures involving the zona pellucida in contemporary ART [25]. In our two cases, it is interesting to note that one was a result of ICSI and embryo biopsy for PGD, while the other was a result of conventional IVF with no zona pellucida manipulation. When looking at the higher order monozygotic pregnancies reported to date, $55 \%(12 / 20)$ were a result of ICSI, $25 \%(5 / 20)$ had assisted hatching and $10 \%(2 / 20)$ underwent embryo biopsy. Again, while the numbers are too small to perform meaningful statistical analysis, it is interesting to note that $2 / 20$ have had embryo biopsy, which is generally a relatively uncommon procedure in most ART units.

There have also been several reports suggesting increased risk of monozygotic twinning with embryo transfers during the blastocyst stage [28-31]. Mechanisms suggested for this association involve extended culture time, culture media composition and laboratory experience [32-35]. However, the majority of the reports suggesting this correlation are limited by the fact that they do not include a cleavage stage embryo transfer group for comparison, more recent analyses have somewhat refuted this correlation. A recent meta-analysis found no increased risk in monozygotic twinning in blastocyst versus cleavage stage transfer, when evaluating ART cycles from 2002 onwards [36]. In addition, a study from the Danish National Cohort project found no increase in overall monozygotic twinning rates when comparing women conceiving naturally versus women conceiving following ART treatment, although they did not perform subgroup analysis for the stage of embryo transfer [10]. In our two presented cases, it is interesting to note that one occurred as a result of a day 4 embryo transfer, whereas the other 
as a result of a blastocyst transfer. Furthermore, when looking at the twenty cases of higher order $(\geq 3)$ monozygotic multiple pregnancies to date, it appears $55 \%$ $(11 / 20)$ have occurred as a result of a blastocyst transfer and $45 \%(9 / 20)$ as a result of cleavage stage transfer (Table 1). Although the numbers are too small to perform meaningful statistical analysis, it can be deduced that currently, there is no significant trend in favour of either stage of embryo transfer.

Another point of interest is that of our two presented cases, the first pregnancy was initially misdiagnosed as a triplet rather than a quadruplet pregnancy until the time when the foetuses were scanned at a later gestational age. In the second case, the diagnosis of twin or triplet pregnancy was suspected at the initial 2D US, but was only clearly confirmed by 3D US, which demonstrated all three yolk sacs and embryos in a single coronal plane. Indeed, making the diagnosis of higher order monozygotic multiple pregnancies with greater confidence at an early stage can be of benefit in the context of ART where the first scan is typically performed at 6 weeks of gestation. This is important as it allows for accurate diagnosis, counselling and referral for appropriate management from very early.

In conclusion, we have presented two cases of monozygotic quadruplet and triplet pregnancies following ART, both of which have undergone successful selective foetal reduction. To our knowledge, the former is the first viable case of monozygotic quadruplet pregnancy in the world, while the latter is the third reported case of a viable MCTA pregnancy following conventional IVF with single ET.

\section{Compliance with ethical standards}

Conflict of interest The authors declare that they have no competing interests.

Open Access This article is distributed under the terms of the Creative Commons Attribution 4.0 International License (http:// creativecommons.org/licenses/by/4.0/), which permits unrestricted use, distribution, and reproduction in any medium, provided you give appropriate credit to the original author(s) and the source, provide a link to the Creative Commons license, and indicate if changes were made.

\section{References}

1. Thurin A, Hausken J, Hillensjo T, Jablonowska B, Pinborg A, Strandell A, et al. Elective single-embryo transfer versus doubleembryo transfer in in vitro fertilization. N Engl J Med. 2004;351: 2392-402.

2. Aston KI, Peterson CM, Carrell DT. Monozygotic twinning associated with assisted reproductive technologies: a review. Reproduction. 2008;136:377-86.

3. Derom C, Vlietinck R, Derom R, Van den Berghe H, Thiery M. Increased monozygotic twinning rate after ovulation induction. Lancet. 1987;1:1236-8.
4. MacGillivray I. Epidemiology of twin pregnancy. Semin Perinatol. 1986;10:4-8.

5. Edwards RG, Mettler L, Walters DE. Identical twins and in vitro fertilization. J In Vitro Fert Embryo Transf. 1986;3(2):114-7.

6. Wenstrom KD, Syrop CH, Hammitt DG, Van Voorhis BJ. Increased risk of monochorionic twinning associated with assisted reproduction. Fertil Steril. 1993;60:510-4.

7. Blickstein I, Jones C, Keith LG. Zygotic-splitting rates after singleembryo transfers in in vitro fertilization. N Engl J Med. 2003;348: 2366-7.

8. Sills ES, Tucker MJ, Palermo GD. Assisted reproductive technologies and monozygous twins: implications for future study and clinical practice. Twin Res. 2000;3:217-23.

9. Alikani M, Cekleniak NA, Walters E, Cohen J. Monozygotic twinning following assisted conception: an analysis of 81 consecutive cases. Hum Reprod. 2003;18:1937-43.

10. Zhu JL, Basso O, Obel C, Christensen K, Olsen J, Danish National Birth C. Infertility, infertility treatment and twinning: the Danish National Birth Cohort. Hum Reprod. 2007;22:1086-90.

11. Pantos K, Kokkali G, Petroutsou K, Lekka K, Malligiannis P, Koratzis A. Monochorionic triplet and monoamniotic twins gestation after intracytoplasmic sperm injection and laser-assisted hatching. Fetal Diagn Ther. 2009;25:144-7.

12. Imaizumi Y. A comparative study of zygotic twinning and triplet rates in eight countries, 1972-1999. J Biosoc Sci. 2003;35:287302.

13. Allen G. A differential method for estimation of type frequencies in triplets and quadruplets. Am J Hum Genet. 1960;12:210-24.

14. Allen G. Quantitative variation in sets of triplets and quadruplets: a simulation. Hum Biol. 1995;67:251-63.

15. Luke B. The changing pattern of multiple births in the United States: maternal and infant characteristics, 1973 and 1990. Obstet Gynecol. 1994;84:101-6.

16. O'Brien BM, Feltovich HM, Carr SR, Luks FI. Feto-fetal transfusion syndrome in monochorionic quadruplets. Obstet Gynecol. 2010;115:470-2.

17. Faber S, Risse S, Steinke H, Thome U, Stepan H. Monochorionic quadruplet pregnancy without severe complications: sonographic work-up and placental findings. Ultrasound Obstet Gynecol. 2013;41:707-8.

18. Timor-Tritsch IE, Fleischer A, Monteagudo A, Valderrama E. Monochorionic quadramniotic quadruplets: sonographic workup. Fetal Diagn Ther. 1997;12:363-7.

19. Bauquis C. The world's number of IVF and ICSI babies has now reached a calculated total of 5 million. In. Brussels: ESHRE, 2012.

20. Liu FH, He L, Long XL, Sun XF, Zhang WH, Zeng XX, et al. Monozygotic quadruplets after in vitro fertilization and embryo transfer. Fertil Steril. 2010;94:2301-2.

21. Alikani M, Noyes N, Cohen J, Rosenwaks Z. Monozygotic twinning in the human is associated with the zona pellucida architecture. Hum Reprod. 1994;9(7):1318-21.

22. Slotnick RN, Ortega JE. Monoamniotic twinning and zona manipulation: a survey of U.S. IVF centers correlating zona manipulation procedures and high-risk twinning frequency. J Assist Reprod Genet. 1996;13(5):381-5.

23. Saito H, Tsutsumi O, Noda Y, Ibuki Y, Hiroi M. Do assisted reproductive technologies have effects on the demography of monozygotic twinning? Fertil Steril. 2000;74(1):178-9.

24. Talansky BE, Gordon JW. Cleavage characteristics of mouse embryos inseminated and cultured after zona pellucida drilling. Gamete Res. 1988;21:277-87.

25. Haimov-Kochman R, Daum H, Lossos F, Aizenman E, Werner M, Yagel S, et al. Monozygotic multiple gestation after intracytoplasmic sperm injection and preimplantation genetic diagnosis. Fertil Steril. 2009;2037(92):e11-7. 
26. Sills ES, Moomjy M, Zaninovic N, Veeck LL, McGee M, Palermo GD, et al. Human zona pellucida micromanipulation and monozygotic twinning frequency after IVF. Hum Reprod. 2000;15:890-5.

27. Elizur SE, Levron J, Shrim A, Sivan E, Dor J, Shulman A. Monozygotic twinning is not associated with zona pellucida micromanipulation procedures but increases with high-order multiple pregnancies. Fertil Steril. 2004;82:500-1.

28. Behr B, Fisch JD, Racowsky C, Miller K, Pool TB, Milki AA. Blastocyst-ET and monozygotic twinning. J Assist Reprod Genet. 2000;17:349-51.

29. Peramo B, Ricciarelli E, Cuadros-Fernandez JM, Huguet E, Hernandez ER. Blastocyst transfer and monozygotic twinning. Fertil Steril. 1999;72:1116-7.

30. Sheiner E, Har-Vardi I, Potashnik G. The potential association between blastocyst transfer and monozygotic twinning. Fertil Steril. 2001;75:217-8.

31. Sheiner E, Kivilevitch Z, Levitas E, Sonin Y, Albotiano S, Har-Vardi I. Monozygotic twins following blastocyst transfer: a report of two cases. Eur J Obstet Gynecol Reprod Biol. 2001;98: $135-8$.

32. Moayeri SE, Behr B, Lathi RB, Westphal LM, Milki AA. Risk of monozygotic twinning with blastocyst transfer decreases over time: an 8-year experience. Fertil Steril. 2007;87:1028-32.

33. da Costa AA, Abdelmassih S, de Oliveira FG, Abdelmassih V, Abdelmassih R, Nagy ZP, et al. Monozygotic twins and transfer at the blastocyst stage after ICSI. Hum Reprod. 2001;16:333-6.

34. Cassuto G, Chavrier M, Menezo Y. Culture conditions and not prolonged culture time are responsible for monozygotic twinning in human in vitro fertilization. Fertil Steril. 2003;80:462-3.

35. Jain JK, Boostanfar R, Slater CC, Francis MM, Paulson RJ. Monozygotic twins and triplets in association with blastocyst transfer. J Assist Reprod Genet. 2004;21:103-7.

36. Chang HJ, Lee JR, Jee BC, Suh CS, Kim SH. Impact of blastocyst transfer on offspring sex ratio and the monozygotic twinning rate: a systematic review and meta-analysis. Fertil Steril. 2009;91:2381-90. 